\title{
Shunt infection and malfunction in patients with myelomeningocele
}

\author{
Michael D. White, MD, Michael M. McDowell, MD, Nitin Agarwal, MD, and Stephanie Greene, MD \\ Department of Neurological Surgery, Children's Hospital of Pittsburgh, University of Pittsburgh Medical Center, Pittsburgh, \\ Pennsylvania
}

\begin{abstract}
OBJECTIVE Myelomeningocele (MMC) is frequently complicated by symptomatic hydrocephalus, necessitating early permanent CSF diversion and revision surgeries. Shunt infections are a common cause of shunt malfunction. This study aims to characterize long-term shunt-related outcomes of patients undergoing MMC closure.
\end{abstract}

METHODS A total of 170 patients undergoing MMC closure between the years of 1995 and 2017 were identified from a retrospective review of a prospectively populated surgical database at the Children's Hospital of Pittsburgh. Patients who underwent MMC closure and required ventriculoperitoneal (VP) shunt insertion met criteria and were included in the primary study analysis. Analysis with a Fisher exact test was performed for categorical variables, and Mann-Whitney U-tests were utilized for numerical data.

RESULTS Of the 158 total patients undergoing MMC closure and meeting inclusion criteria, 137 (87\%) required VP shunt insertion. These 137 patients demonstrated a shunt revision rate of $21.1 \%$ per person-year and a shunt infection rate of $2.1 \%$ per person-year over a mean follow-up of 10.8 years. Patients had a mean of $3.4 \pm 0.6$ shunt surgeries prior to their first infection. Patients undergoing immediate shunt removal, external ventricular drain placement, or shunt replacement after clearing the infection had lower rates of subsequent infections than patients who initially were managed with shunt externalization $(p<0.001)$. Placement of a shunt at the time of MMC closure was not found to be a risk factor for infection. Of patients with initial shunt placement after the implementation of the Hydrocephalus Clinical Research Network protocol in 2011, the authors' institution has had a shunt infection rate of $4.2 \%$ per person-year and a revision rate of $35.7 \%$ per person-year.

CONCLUSIONS This study describes long-term outcomes of shunted MMC patients and factors associated with shunt infections. Most patients underwent multiple revisions prior to the first shunt infection. Shunt externalization may be ineffective at clearing the infection and should be avoided in favor of early shunt removal and external ventricular drainage, followed by shunt replacement once infection is demonstrated to have cleared.

https://thejns.org/doi/abs/10.3171/2020.9.PEDS20313

KEYWORDS myelomeningocele; spina bifida; shunt infection; ventriculoperitoneal shunt; hydrocephalus

$\mathrm{M}$ yelomeningocele (MMC), the most common neural tube defect, is complicated by hydrocephalus in $40 \%-90 \%$ of cases. Previous studies have demonstrated that 50\%-96\% of patients with MMC undergo ventriculoperitoneal (VP) shunt placement. ${ }^{1-4}$ Among pediatric patients with hydrocephalus, there is a historically high rate of shunt failure and need for revision surgeries, reported to be approximately $33 \% .{ }^{5}$ However, revision rates over a child's lifetime are thought to be much higher, and studies investigating long-term outcomes have reported that $79 \%-84 \%$ of all hydrocephalus patients require at least one revision. ${ }^{6-9}$ This number is equally high in patients with MMC among studies with long-term follow-up..$^{10,11}$ Reported rates of shunt failure are dependent on the length of follow-up, and there is a paucity of studies on MMC patients with a follow-up of more than 10 years; it is logical to conclude that the actual percentages are likely higher across a patient's lifetime.

With the frequent need for VP shunt placement in the MMC population, shunt infections are a significant source of morbidity for these patients. The association between MMC and the development of shunt infection remains inconclusive within the current literature. Additionally, longterm shunt infection rates for MMC patients with modern management strategies are also unclear, with older studies reporting a range of $19 \%-23 \% .^{10,11}$ However, there is a

ABBREVIATIONS ETV = endoscopic third ventriculostomy; EVD = external ventricular drain; HCRN = Hydrocephalus Clinical Research Network; MMC = myelomeningocele; $\mathrm{VP}=$ ventriculoperitoneal; $\mathrm{WBC}=$ white blood cell count.

SUBMITTED April 20, 2020. ACCEPTED September 3, 2020.

INCLUDE WHEN CITING Published online February 26, 2021; DOI: 10.3171/2020.9.PEDS20313. 
paucity of data on long-term shunt outcomes among this population, and further investigation is imperative to improving management of MMC-related hydrocephalus. In this retrospective institutional 22-year cohort analysis, we sought to characterize long-term rates of shunt malfunction and infection among MMC patients.

\section{Methods}

We retrospectively reviewed the charts of 170 patients undergoing closure of MMC from 1995 to 2017 from an operative database maintained by the neurosurgery department at the Children's Hospital of Pittsburgh. ${ }^{12}$ Among the 170 identified patients, 86 were males $(50.58 \%)$ and 84 were females $(49.41 \%)$. Patients with myelocystocele $(\mathrm{n}=$ 1) or meningocele $(n=3)$ and those receiving initial care at another hospital $(\mathrm{n}=3)$ were excluded. Patients who succumbed to perinatal death $(\mathrm{n}=5)$ were also excluded, leaving 158 patients for analysis. Of the $158 \mathrm{MMC}$ patients identified, 137 (87\%) required a VP shunt. Twenty-one patients $(13 \%)$ did not require shunting and were excluded from further analysis, with 2 of these patients being successfully treated with endoscopic third ventriculostomy (ETV). All patients included in the study underwent MMC closure and CSF diversion with a VP shunt at this institution. The diagnosis of hydrocephalus was made by identifying a rapidly increasing head circumference crossing above the 97th percentile for gestational age and progressive ventriculomegaly on serial ultrasound. These findings were often accompanied by splaying of sutures, bulging of the anterior fontanel, or pseudomeningocele formation at the site of MMC repair; however, these findings alone were insufficient to diagnose hydrocephalus in the absence of ventriculomegaly and macrocephaly. All patients were followed in our institution's multidisciplinary spina bifida clinic, which includes neurosurgery, orthopedics, urology, and physical medicine and rehabilitation physicians, and physical, occupational, and speech therapists. VP shunt infections were defined as positive CSF cultures or elevated CSF white blood cell counts (WBCs) $(>1$ white/300 red blood cells). Shunt malfunction was defined as any instance in which a patient with an indwelling CSF shunt required operative shunt revision or replacement for worsening hydrocephalus symptomatology, often accompanied by sluggish flow observed in a nuclear medicine shunt flow study or enlarging ventricles on imaging.

In the beginning of 2011, the Children's Hospital of Pittsburgh implemented the Hydrocephalus Clinical Research Network (HCRN) protocol for all shunt procedures in an effort to reduce the incidence of shunt infections. This operative protocol includes checklist items, such as administering preoperative and postoperative antibiotics, double gloving by all participants, positioning the patient's head away from the main operating room door, and using Ioban drapes $(3 \mathrm{M})$. Comparisons were made between patients who received their first shunt insertion prior to 2011 (pre-2011 group) and those who received their first shunt insertion during/after 2011 (post-2011 group). At our institution, the implementation of the HCRN protocol resulted in significant changes to our practice. First, antibiotic-impregnated catheters were not routinely used prior to this change, with the exception of after an infection, and these were consistently used according to the HCRN protocol after 2011. Second, the courses of perioperative antibiotics prior to the HCRN protocol were variable; however, after the protocol implementation every patient received 24 hours of a cephalosporin as first line. Additionally, prior to 2011, surgical antiseptics such as Avagard were used regularly as opposed to a full scrub after the first case of the day; however, a full scrub was consistently used for every case after the HCRN implementation. Finally, there was routine use of double gloving for all participants during all shunt procedures.

Analysis with a Fisher exact test was performed for categorical variables, and a Mann-Whitney U-test was utilized for numerical data. Linear regression was performed for age at first shunt placement and revision rates. All analysis was performed using GraphPad Prism 7.0 software.

\section{Results}

Among the 158 patients undergoing closure of MMC and included for analysis, $137(87 \%)$ required VP shunt placement for treatment of hydrocephalus. Of the $137 \mathrm{pa}-$ tients requiring shunt placement, 98 (72\%) required at least one revision. The overall shunt revision rate was $21.1 \%$ per person-year, with a median rate of 0.14 revisions per year. There was a mean of 2.28 revisions per patient, ranging from 0 to 23 revisions. Over the study period, the percentage of patients receiving shunts changed over time: $89.5 \%$ (120/134) received shunts prior to 2012 compared to $75 \%$ (18/24) after 2012, and 90.1\% (127/141) prior to 2014 compared to $64.7 \%$ (11/17) after 2014. The mean follow-up for included patients was $130 \pm 71$ months (10.8 years).

The overall infection rate for shunted patients was $2.1 \%$ per person-year, with $15.3 \%$ of the cohort developing at least 1 infection over a mean of 10.8 years of follow-up. Shunt infection was the indication for 32 surgeries (10.3\% of all shunt revisions) during the study period. The mean and median ages of first infection were $30.6 \pm 9.6$ months and 3.5 months, respectively, with over half (52\%) of initial infections occurring within the 1st year of life. A mean of $3.4 \pm 0.6$ shunt surgeries (including those for initial placement and revisions) were performed before the development of a patient's first shunt infection. The mean time from last shunt surgery to the development of shunt infection was $2.0 \pm 5.8$ months, with a range of $0.07-11.0$ months. There were 2 outliers in terms of mean time from last shunt surgery that were not counted in this range: the first was a shunt infection that occurred 49.3 months after the last shunt surgery but was secondary to an adjacent cellulitis. The infection seemingly resolved with local care over 4 days and without antibiotics, but 10 days later the patient developed scabbing and pus overlying her shunt tubing. The second outlier was a shunt infection due to an infected incision occurring shortly after a craniosynostosis procedure. These 2 cases were not considered postoperative shunt infections after a recent shunt surgery and were not calculated into the range. Among patients developing a shunt infection, 7 (33\%) developed subsequent shunt infections. The overall 6-month reinfection rate was $28.6 \%$, compared to $14.8 \%$ of the overall pediatric population as 
reported in a previous HCRN collaborative study (relative risk 2.2, $\mathrm{p}=0.02) .{ }^{13}$ Four patients $(19 \%)$ in the infection group had a total of 3 infections each over an average of 62 months. One of the 4 patients required plastic surgery to assist with wound closure with the first infection, and none of these 4 patients required further plastic surgery. The mean and median times until repeated infection were 27.5 \pm 10.9 months and 6.4 months, respectively, with a mean of $1.8 \pm 0.5$ revisions between infections. In the setting of shunt infection, 23 cases (72\%) were associated with visible wound complications, including skin breakdown, swelling, CSF leakage, purulent drainage, and erythema. Shunt hardware was visibly exposed on presentation in 8 cases $(25 \%)$ of patients developing infection. Elevated serum WBCs were found in $46 \%$ of infections, and fever was noted in $30 \%$ of infections (serum WBCs and fever were not documented in 5 of the 32 infections). Shunt infection statistics are shown in Table 1.

Of patients with shunt infections, $26(81 \%)$ underwent removal of the entire shunt system and placement of an external ventricular drain (EVD), followed by replacement at a later date, whereas 6 patients $(19 \%)$ had distal externalization of the shunt. Of these 6 externalizations, 3 were later reinserted with replacement of the distal catheter and 3 were later completely removed and replaced. Patient age at the time of infection ( $\mathrm{p}=0.49)$, causative organism ( $\mathrm{p}=$ 0.12 ), time since last shunt surgery $(p=0.43)$, and whether the treatment modality was for initial infection versus subsequent infection $(p=0.39)$ were compared between the externalization and removal groups, with no significant differences being found. The mean time until reinsertion of externalized shunts was 5 days. Five of the 6 patients (83\%) whose shunts were initially externalized developed future shunt infections, compared to only 5 of the 26 patients $(19 \%)$ who were not externalized. There was a mean time to reinfection of 30.6 months for externalized shunts and a mean of 2.6 revisions between infections. Patients who underwent externalization of their shunt were found to be more likely to develop a subsequent infection compared to those who had removal of the entire shunt system ( $p<0.0001)$. However, all cases of subsequent infection were found to have a different causative organism than the previous infection.

Of the 21 patients receiving an EVD at the time of MMC closure, 2 patients (9.5\%) had shunt infection as the indication for their first revision, while 4 patients (3.6\%) without EVD placement at the time of closure had shunt infection as the indication for their first revision $(p=0.23)$. For the 26 patients receiving a VP shunt at the time of closure, no patients had shunt infection as the indication for their first revision, with 7 (6.3\%) of those without VP shunt placement at closure having infection as the indication for their first revision $(\mathrm{p}=0.35)$. Only 2 of the patients with a VP shunt placed at the time of closure were found to have infection during any point in the study. Among these 2 patients, there were a total of 4 shunt infections, with only 1 infection occurring before the age of 8 years (at 2.5 months). Three of these 4 infections were caused by skin flora (1 Staphylococcus epidermidis and 2 Cutibacterium acnes). Overall, patients with a shunt placed at the time of closure were not at higher risk for either S. epider-
TABLE 1. Infection statistics

\begin{tabular}{lc}
\hline & Value \\
\hline Infection rate per person-yr & $2.1 \%$ \\
\hline No. of patients w/ $\geq 1$ infection & $21(15.3 \%)$ \\
\hline Age at 1st infection, mos & $3.5(30.6 \pm 9.6)$ \\
\hline Initial infections in 1st year of life & $11(52 \%)$ \\
\hline No. of patients w/ multiple infections & $7(33 \%)$ \\
\hline Time to reinfection, mos & $3.0(27.5 \pm 10.9)$ \\
\hline No. of revisions between infections & $1.8 \pm 0.5$ \\
\hline 6-mo reinfection rate & $28.6 \%$ \\
\hline No. of shunt surgeries before 1st infection & $3.4 \pm 0.6$ \\
\hline Time from last shunt surgery to infection, mos & $2.0 \pm 5.8$ \\
\hline Infectious symptoms on presentation & $72 \%$ \\
\hline Visible wound complications & $25 \%$ \\
\hline Shunt hardware visibly exposed & $46 \%$ \\
\hline Elevated serum WBC & $30 \%$ \\
\hline Fever & \\
\hline
\end{tabular}

Six-month reinfection rates among our MMC cohort were significantly higher than those of the general pediatric hydrocephalus population according to previous HCRN reports (relative risk 2.2, $p=0.02$ ). ${ }^{20}$

Values are presented as number (\%) of patients, mean $\pm \mathrm{SD}$, or median (mean \pm SD) unless otherwise indicated.

midis or $C$. acnes infections $(\mathrm{p}=0.30)$. However, among all patients developing a shunt infection, there was a trend of having their first shunt placed sooner after birth, at a mean of 10.29 days after birth, compared to 24.34 days after birth for patients not developing shunt infections; this trend was not statistically significant. There was also no significant trend observed for age at first shunt placement and revision rates $\left(\mathrm{r}^{2}<0.01, \mathrm{p}=0.89\right)$.

At our institution ETV has not been widely used for the management of MMC patients, with 9 patients receiving ETV during the study period. Only 2 patients (22\%) have been successfully treated with ETV and did not require a shunt for hydrocephalus. Of the 9 patients, ETV was performed in 4 patients prior to receiving a shunt, 2 at the time of shunt placement, and 3 after initial shunt placement. Choroid plexus cauterizations are another alternative to treatment with a VP shunt; however, this procedure is not extensively performed at our institution.

\section{Impact of HCRN Shunt Protocol on Infection Rates}

Revision and infection rates for patients with initial shunt placement before 2011 (pre-2011 group) and during/after 2011 (post-2011 group) were compared (Table 2 ). There were no statistically significant differences between the two time periods for any comparison. The revision rates per person-year for the pre-2011 and post2011 groups were $19.9 \%$ and $34.7 \%$, respectively. Additionally, the infection rates per person-year for the same groups were $2.0 \%$ and $4.2 \%$, respectively. The mean and median ages at first infection for patients in the pre-2011 group were $30.5 \pm 11.8$ months and 2.9 months, respectively, compared to $27.2 \pm 8.8$ months and 33.9 months for the post-2011 group. The initial infections for patients 
TABLE 2. Comparisons of the pre-2011 cohort and the post-2011 cohort (after implementation of the HCRN protocol)

\begin{tabular}{lccc}
\hline & Pre-2011 & Post-2011 & p Value \\
\hline $\begin{array}{l}\text { Shunt revision rate per } \\
\text { person-yr }\end{array}$ & $19.9 \%$ & $34.7 \%$ & 0.45 \\
\hline $\begin{array}{l}\text { Shunt infection rate per } \\
\text { person-yr }\end{array}$ & $2.0 \%$ & $4.2 \%$ & 0.47 \\
\hline Age at 1st infection, mo & $2.9(30.5 \pm 11.8)$ & $33.9(27.2 \pm 8.8)$ & 0.32 \\
\hline $\begin{array}{l}\text { Mean no. of revisions } \\
\text { prior to 1st infection }\end{array}$ & $3.5 \pm 0.7$ & $3.5 \pm 1.0$ & 0.37 \\
\hline
\end{tabular}

Values are presented as median (mean $\pm S D$ ) or mean \pm SD unless otherwise indicated. None of the differences between groups reached statistical significance.

in the pre-2011 group had a mean of $3.5 \pm 0.7$ prior revisions, whereas initial infections in the post-2011 group had a mean of $3.5 \pm 1.0$ prior revisions. Finally, the time since last shunt surgery for each infection was stratified into five groups: less than 1 week, 1 week to 1 month, 1 to 6 months, 6 to 12 months, and greater than 12 months. Comparison between the pre-2011 and post-2011 groups did not show any statistically significant difference. ${ }^{14}$

\section{Microbiological Analysis}

Microbiological testing of CSF and shunt components revealed an infectious organism in $75 \%$ of infections. $S$. epidermidis and $C$. acnes were the two most common infectious organisms, comprising $35 \%$ and $23 \%$, respectively, of all identified bacteria. There was no significant difference in the causative pathological organisms in infections occurring before 2011 versus in 2011 and beyond $(\mathrm{p}=0.7527)$. The causative pathogens are represented in Table 3. In almost all patients who developed more than one infection, the pathogen identified in the later infection was different than that found in the previous infections. The single exception was 1 patient who had 2 infections with positive cultures for $C$. acnes separated by more than 9.5 years.

Differing presentations between patients with infections due to gram-negative rods versus $S$. epidermidis or $C$. acnes were compared. Infections due to gram-negative rods were found to occur at younger patient ages compared with those with $S$. epidermidis or C. acnes ( $\mathrm{p}=$ 0.032 ), with mean ages of presentation at 1.67 months and 67.16 months, respectively. The mean and median times since last shunt surgery were 0.77 months and 1.13 months for gram-negative rods and 3.53 months and 0.96 months for $S$. epidermidis and C. acnes, respectively $(\mathrm{p}=0.81)$. Patients with infections secondary to $S$. epidermidis or $C$. acnes were more likely to present with wound breakdown and complications (80\% of patients) compared to patients with gram-negative rod infections $(66 \%$ of patients); however, this trend was not statistically significant $(p=0.55)$. Rates of fever at the time of presentation for patients with gram-negative rod infections versus $C$. acnes or S. epidermidis were $66 \%$ and $25 \%$, respectively $(\mathrm{p}=$ $0.24)$. There were 2 patients with $C$. acnes infections who did not originally present with wound breakdown. Among
TABLE 3. Pathogenic organisms identified in the shunt infections

\begin{tabular}{lccc}
\hline \multicolumn{1}{c}{ Organism } & Total & $\begin{array}{c}\text { Pre-2011 } \\
\text { Group }\end{array}$ & $\begin{array}{c}\text { Post-2011 } \\
\text { Group }\end{array}$ \\
\hline Staphylococcus epidermidis & 9 & 8 & 1 \\
\hline Cutibacterium acnes & 6 & 6 & 0 \\
\hline Staphylococcus aureus & 4 & 3 & 1 \\
\hline Candida albicans & 2 & 1 & 1 \\
\hline Pseudomonas aeruginosa & 1 & 1 & 0 \\
\hline Enterobacter cloacae & 1 & 1 & 0 \\
\hline Haemophilus influenzae & 1 & 1 & 0 \\
\hline Alpha-hemolytic streptococci & 1 & 1 & 0 \\
\hline Gram-negative rods, no speciation & 1 & 1 & 0 \\
\hline Negative cultures & 8 & 6 & 2 \\
\hline Total & 34 & 29 & 5 \\
\hline
\end{tabular}

In 34 total infections, 26 pathogenic organisms ( 9 separate identified organisms) were identified in 32 infections. Two patients had multiple organisms identified on bacterial culture. One patient in the pre-2011 group had a CSF culture that grew $C$. acnes and a catheter tip culture that grew $S$. epidermidis. The second patient was in the post-2011 group and grew S. epidermidis in the CSF and blood, with $S$. aureus also positive on CSF culture.

these 2 cases, one patient had a fever of unknown origin in which further workup revealed a urinary tract infection as well as C.acnes on CSF culture, whereas the other patient presented with 2 days of vomiting, headache, and fatigue, with workup demonstrating an abdominal abscess and subsequently positive CSF cultures. There was 1 patient with infection secondary to $S$. epidermidis who did not present with wound breakdown, but rather headache and upward gaze palsy, and a positive CSF culture found incidentally during revision.

In 8 patients (25\%), infections yielded negative CSF and blood cultures on infectious workup. All but 1 case $(87.5 \%)$ presented with wound breakdown, erythema, and/ or drainage. Three of the 8 patients (38\%) were already on antibiotics at the time of presentation, which likely influenced culture results. These 3 patients all presented with wound breakdown, 1 patient with frank pus from the incision site and elevated WBC on CSF analysis. Another patient presented with wound breakdown, peripheral leukocytosis, and elevated WBC on CSF analysis. One patient presented with fluid leakage from the incision site, pseudocyst within the abdomen, peripheral leukocytosis, and elevated WBC in the CSF. The 1 patient without wound breakdown presented with lethargy and irritability, and further workup demonstrating elevated WBC, low glucose, and high protein on CSF analysis. The final 2 patients presented with wound breakdown and elevated WBC on CSF analysis.

\section{Discussion}

In this study, one of the largest single-institution studies to date of shunt infections in MMC patients, we demonstrated the potential complications of shunt externalization versus complete removal and investigated long-term outcomes at a median time exceeding 10 years. The longterm institutional rate of shunt revisions among MMC 
patients was $21.1 \%$ per person-year, with $72 \%$ of patients in the cohort requiring at least one revision. These rates are comparable to those previously reported in the literature..$^{10,11,15,16}$ Of patients included in the study, $84 \%$ had at least 5 years of follow-up and 54\% of patients had at least 10 years of follow-up. The range of follow-up was 1-265 months, with only 3 patients having less than 1 year of follow-up.

MMC patients historically have higher rates of shunt infections than the general pediatric hydrocephalus population. Previous studies investigating shunt infections in all hydrocephalus patients have reported shunt infection rates ranging from $3 \%$ to $11 \%$ within 2 years of insertion, ${ }^{17-20}$ and higher rates of $8 \%-13.6 \%$ in studies with at least 7 years of follow-up. ${ }^{6,21,22}$ These findings contrast with those in MMC patients, for whom shunt infections have been shown to occur at higher rates of $13.5 \%-23 \%,{ }^{10,11,22-25}$ with infection rates on the higher end of that range (19\%-23\%) among studies with at least 9 years of follow-up. ${ }^{10,11}$ The observed shunt infection rate for the current study was $2.1 \%$ per person-year, with $15.3 \%$ of the cohort developing at least 1 infection over the mean follow-up of 10.8 years. This infection rate is on the lower end of the infection rates within the literature. Of note, more than half of infections (52\%) occurred within the 1st year of life (also within 1 year of shunt placement). Prior studies have demonstrated a similar timeline in overall shunt revisions, with most occurring within the 1st year. ${ }^{16}$ Most infections (88\%) also occurred less than 6 months after a previous shunt surgery. Only $9 \%$ of infections were found to have occurred more than 1 year after prior shunt surgery, a rate that is relatively consistent with other reports of $12 \% .5,22,26$ The use of ETV may play a role in reducing infection rates in these patients; however, the low number of ETV attempts performed in the present study is a limitation in assessing this relationship.

All cases of shunt infections during the study period were effectively treated with antibiotics and deemed cleared by the institution's infectious disease service prior to shunt replacement, with no observed deaths secondary to shunt infection. Among patients who developed multiple shunt infections, the causative organisms of later infections were found to be different from those causing the prior infection in almost all cases. There may be a predisposition to shunt infection in these patients, with thin or tenuous skin from body habitus or multiple operations, behavioral issues common in pediatric patients such as scratching or picking at healing wounds, or poor nutrition resulting in a compromised immune system. Of note, almost all (6 of 7) patients with multiple shunt infections had a previous infection with some aspect of visible wound complication, including purulent drainage, CSF leakage, erythema, or wound breakdown, though only 1 patient required plastic surgery involvement.

\section{Shunt Externalization}

Patients who underwent shunt externalization in the setting of infection, as opposed to immediate removal of the shunt and placement of an EVD, had significantly higher rates of subsequent infection. There is no standard of care at present in terms of operative management of shunt infection regarding shunt externalization versus shunt removal. One study by Simon et al. demonstrated trends of higher reinfection rates among patients undergoing initial externalization (22\%) compared to initial shunt removal and EVD placement (15\%) in the ensuing 6 months after treatment, although these differences did not reach statistical significance. ${ }^{13}$ This study did not include the causative organisms of the infections, so it is unknown if the subsequent infections represented true reinfections with the same pathogen. Another study demonstrated the opposite trend, with a lower cumulative risk of reinfection with initial externalization (20\%) compared to initial shunt removal (27\%) within 6 months posttreatment, but again did not reach statistical significance. ${ }^{27}$ The present study demonstrated a significantly higher rate of subsequent infections in patients with initial shunt externalization in comparison to those with shunt removal and EVD placement. Externalization may allow for the foreign body of the shunt to persistently serve as a nidus for infection that is impenetrable to antibiotics. However, given that none of the subsequent infections were caused by the same organism as the initial infection, the violation of the skin at the externalization point would more likely explain the higher rate of subsequent infections, especially given that all of the identified causative organisms from the subsequent infections were from skin flora (S. epidermidis, C. acnes, or group A streptococci). At our institution, we externalize at the clavicle and then perform a revision at the site of the shunt valve, replacing the tubing and retunneling. Instead, it may be better to perform complete removal and placement on the other side when possible, even if the externalization is only temporary. Our data show that initial externalization of shunts after infection may result in a higher risk for subsequent infections, as opposed to immediate removal and EVD placement with delayed replacement of the shunt system. The superiority of one technique over the other is unclear in the context of the current literature, and further study of reinfection rates between different management options and the timing of reinternalization of externalized shunts may be considered.

\section{CSF Diversion at Closure}

A previous study investigated temporary CSF diversion with EVD or VP shunt placement at the time of MMC closure in patients with evidence of hydrocephalus at birth and found that this procedure reduces the rate of wound complications in MMC closure. ${ }^{28}$ Given the relatively high risk of developing shunt failure and infection in MMC patients, it is important to explore any potential correlation between early CSF diversion at the time of closure and the presence of shunt infection. The present study shows that CSF diversion with either an EVD or a VP shunt at closure was not significantly associated with the first revision resulting from infection. These results indicate that CSF diversion at the time of closure reduces the risk of MMC wound complications without a significant increase in the risk of shunt infection.

\section{HCRN Protocol}

In 2011, the Hydrocephalus Clinical Research Network 
(HCRN) reported a study of a new standardized surgical protocol in an effort to reduce the incidence of VP shunt infections. The HCRN protocol was implemented in four different centers, with three of the centers showing significantly reduced rates of shunt infections and the remaining center not showing any change. Overall, the protocol demonstrated a significant reduction in VP shunt infection rates. ${ }^{14}$ Our institution adopted the HCRN protocol in 2011 as a standard practice for all VP shunt insertions and revisions. In the present study, analysis of shunt infections per person-year for children with first shunt insertion before 2011 versus during/after 2011 did not demonstrate a significant difference. Additionally, no difference was observed between the two groups in age at first infection, revisions per year, time from last shunt surgery to infection, and number of revisions before the first infection. Differences in infection rates between these two groups may not be apparent in the current study, due to the lower population size of the group receiving their first shunt insertion during/after 2011 (107 patients before 2011 vs 30 patients during/after 2011). The group receiving shunt insertions during/after $2011 \mathrm{had}$ a shorter length of follow-up, and since it is more common for shunt infections to arise earlier in life, the presence of one or more early infections can skew the infections per person-year values. Additionally, the HCRN study showed a reduction in 6-month infection rates, whereas our study looked at longer-term rates of infections.

\section{Study Limitations}

This study has several limitations, including its retrospective nature. Furthermore, there were a multitude of different practicing neurosurgeons at our institution over the study period with variations in practice preferences. Data on the use of antibiotic-impregnated catheters is not documented in our institution's electronic health records in a manner consistent enough to allow us to analyze this information and its effects on shunt infections. Finally, the generalizability of our findings is limited by the singleinstitutional nature of the study.

\section{Conclusions}

In this study we characterized long-term rates of VP shunt malfunction and infection in MMC patients. In concordance with previous reports, a majority of patients undergoing closure of MMC required CSF diversion and exhibited high rates of shunt malfunction and infection. It was found that initial shunt externalization for infection, as opposed to immediate removal of the shunt system and external drainage, was associated with a significantly higher rate of subsequent infection. Shunt infections can also result from adjacent infections as well as from previous shunt surgeries.

\section{References}

1. Phillips BC, Gelsomino M, Pownall AL, et al. Predictors of the need for cerebrospinal fluid diversion in patients with myelomeningocele. J Neurosurg Pediatr. 2014;14(2):167-172.

2. Marreiros H, Loff C, Calado E. Who needs surgery for pediatric myelomeningocele? A retrospective study and literature review. J Spinal Cord Med. 2015;38(5):626-640.
3. Kim I, Hopson B, Aban I, et al. Treated hydrocephalus in individuals with myelomeningocele in the National Spina Bifida Patient Registry. J Neurosurg Pediatr. 2018;22(6): 646-651.

4. Adzick NS, Thom EA, Spong CY, et al. A randomized trial of prenatal versus postnatal repair of myelomeningocele. $N$ Engl J Med. 2011;364(11):993-1004.

5. Riva-Cambrin J, Kestle JR, Holubkov R, et al. Risk factors for shunt malfunction in pediatric hydrocephalus: a multicenter prospective cohort study. J Neurosurg Pediatr. 2016; 17(4):382-390.

6. Paulsen AH, Lundar T, Lindegaard KF. Pediatric hydrocephalus: 40-year outcomes in 128 hydrocephalic patients treated with shunts during childhood. Assessment of surgical outcome, work participation, and health-related quality of life. $J$ Neurosurg Pediatr. 2015;16(6):633-641.

7. Stone JJ, Walker CT, Jacobson M, et al. Revision rate of pediatric ventriculoperitoneal shunts after 15 years. J Neurosurg Pediatr. 2013;11(1):15-19.

8. Reddy GK, Bollam P, Caldito G. Long-term outcomes of ventriculoperitoneal shunt surgery in patients with hydrocephalus. World Neurosurg. 2014;81(2):404-410.

9. Tervonen J, Leinonen V, Jääskeläinen JE, et al. Rate and risk factors for shunt revision in pediatric patients with hydrocephalus - a population-based study. World Neurosurg. 2017; 101:615-622.

10. Bowman RM, McLone DG, Grant JA, et al. Spina bifida outcome: a 25-year prospective. Pediatr Neurosurg. 2001;34(3): $114-120$

11. Talamonti G, D'Aliberti G, Collice M. Myelomeningocele: long-term neurosurgical treatment and follow-up in 202 patients. J Neurosurg. 2007;107(5)(suppl):368-386.

12. Kellogg R, Lee P, Deibert CP, et al. Twenty years' experience with myelomeningocele management at a single institution: lessons learned. J Neurosurg Pediatr. 2018;22(4):439-443.

13. Simon TD, Hall M, Dean JM, et al. Reinfection following initial cerebrospinal fluid shunt infection. J Neurosurg Pediatr. 2010;6(3):277-285.

14. Kestle JR, Riva-Cambrin J, Wellons JC III, et al. A standardized protocol to reduce cerebrospinal fluid shunt infection: the Hydrocephalus Clinical Research Network Quality Improvement Initiative. J Neurosurg Pediatr. 2011;8(1):22-29.

15. Tuli S, Drake J, Lamberti-Pasculli M. Long-term outcome of hydrocephalus management in myelomeningoceles. Childs Nerv Syst. 2003;19(5-6):286-291.

16. Dupepe EB, Hopson B, Johnston JM, et al. Rate of shunt revision as a function of age in patients with shunted hydrocephalus due to myelomeningocele. Neurosurg Focus. 2016;41(5):E6.

17. Simon TD, Hall M, Riva-Cambrin J, et al. Infection rates following initial cerebrospinal fluid shunt placement across pediatric hospitals in the United States. Clinical article. $J$ Neurosurg Pediatr. 2009;4(2):156-165.

18. James G, Hartley JC, Morgan RD, Ternier J. Effect of introduction of antibiotic-impregnated shunt catheters on cerebrospinal fluid shunt infection in children: a large single-center retrospective study. J Neurosurg Pediatr. 2014;13(1):101-106.

19. Omrani O, O’Connor J, Hartley J, James G. Effect of introduction of a standardised peri-operative protocol on CSF shunt infection rate: a single-centre cohort study of 809 procedures. Childs Nerv Syst. 2018;34(12):2407-2414.

20. van Lindert EJ, Bilsen MV, Flier MV, et al. Topical vancomycin reduces the cerebrospinal fluid shunt infection rate: A retrospective cohort study. PLoS One. 2018;13(1):e0190249.

21. Beuriat PA, Puget S, Cinalli G, et al. Hydrocephalus treatment in children: long-term outcome in 975 consecutive patients. J Neurosurg Pediatr. 2017;20(1):10-18.

22. Vinchon M, Dhellemmes P. Cerebrospinal fluid shunt infection: risk factors and long-term follow-up. Childs Nerv Syst. 2006;22(7):692-697. 
23. Radmanesh F, Nejat F, El Khashab M, et al. Shunt complications in children with myelomeningocele: effect of timing of shunt placement. J Neurosurg Pediatr. 2009;3(6):516-520.

24. Habibi Z, Ertiaei A, Nikdad MS, et al. Predicting ventriculoperitoneal shunt infection in children with hydrocephalus using artificial neural network. Childs Nerv Syst. 2016;32(11): 2143-2151.

25. Stark GD, Drummond MB, Poneprasert S, Robarts FH. Primary ventriculo-peritoneal shunts in treatment of hydrocephalus associated with myelomeningocele. Arch Dis Child. 1974;49(2):112-117.

26. Lee JK, Seok JY, Lee JH, et al. Incidence and risk factors of ventriculoperitoneal shunt infections in children: a study of 333 consecutive shunts in 6 years. J Korean Med Sci. 2012; 27(12):1563-1568.

27. Kulkarni AV, Rabin D, Lamberti-Pasculli M, Drake JM. Repeat cerebrospinal fluid shunt infection in children. Pediatr Neurosurg. 2001;35(2):66-71.

28. McDowell MM, Lee PS, Foster KA, Greene S. The use of external ventricular drainage to reduce the frequency of wound complications in myelomeningocele closure. Pediatr Neurosurg. 2018;53(2):100-107.

\section{Disclosures}

Dr. Agarwal reports receiving royalties from Thieme Medical Publishers.

\section{Author Contributions}

Conception and design: Greene. Acquisition of data: White. Analysis and interpretation of data: White. Drafting the article: White. Critically revising the article: McDowell, Agarwal, Greene.

Reviewed submitted version of manuscript: all authors. Statistical analysis: McDowell, White. Study supervision: Greene.

\section{Correspondence}

Michael M. McDowell: University of Pittsburgh Medical Center, Pittsburgh, PA. mcdowellmm2@upmc.edu. 